\title{
Los Determinantes socio-económicos del delito en España
}

\author{
Antonio Rodríguez Andrés iD \\ Estudiante de doctorado \\ Departamento de Economía, University of Southern Denmark
}

Resumen:

Este trabajo examina, mediante el uso de modelos econométricos de datos de panel, los determinantes socio-económicos del delito en España. Los resultados del estudio sugieren que una vez controlada la posible endogeneidad de la probabilidad de captura, la variable no es significativa. Factores demográficos tienen un impacto sobre las tasas de delicuencia. También se encuentra evidencia empírica de que la renta per capita y el nivel educativo tienen un efecto positivo sobre los niveles de criminalidad mientras que la tasa de desempleo no es un factor significativo.

Palabras clave: delito, datos de panel, endogeneidad

\begin{abstract}
:
This paper studies the determinants of crime for 16 regions from 1994 to 2001 in Spain. A panel data technique is used, where crime rates are related to some deterrence, economic as well as social and demographic variables. Accounting for the endogeneity of the clear up rate renders the probability of punishment insignificant in deterring crime. However, demographic variables appear to have a significant effect on regional crime rates. Additionally, per capita income has a significant effect on overall crime rates, but unemployment is not an important deterrent of crime.
\end{abstract}

Keywords: crime, panel data, endogeneity

Revista Española de Investigación Criminológica REIC AC 01-03 http:www.criminologia.net/revista https://doi.org/10.46381/reic.v1i0.5 


\section{Introducción}

En los últimos años hemos visto como ha crecido la preocupación por el tema de la delincuencia en España. De acuerdo con una encuesta del año 2003, realizada por el Centro de Investigaciones Sociológicas (CIS), un 31.2\% de los españoles considera que la delincuencia es uno de los principales problemas en nuestro país junto con el terrorismo, $46.5 \%$ y el desempleo, $60.5 \%{ }^{1}$. Esto unido al aumento vertiginoso en el número de inmigrantes y su amplia cobertura ofrecida en los medios de comunicación españoles, ha generado un sentimiento popular de que el aumento de la delincuencia tiene su origen en la emigración ilegal. En otra encuesta del año 2003, se ofrecía un dato preocupante, un $44.2 \%$ de los españoles afirma que la inmigración esta favoreciendo el incremento en la inseguridad ciudadana ${ }^{2}$.

Para hacer frente a esta situación, recientemente, el gobierno español ha adoptado un plan de choque contra la delincuencia y la inmigración ilegal ${ }^{3}$. De lo expuesto anteriormente, parece lógico plantearse cuales son los determinantes del delito en España y si verdaderamente la actividad delictiva está ligada a la creciente presencia de inmigrantes en nuestro país. Este es el objetivo del presente trabajo.

En la literatura económica, el trabajo pionero de Becker (1968), ejerció una gran influencia sobre los economistas ${ }^{4}$. Años mas tarde, Ehrlich (1973) presentó por primera vez evidencia empírica del modelo económico del crimen utilizando datos de los Estados Unidos. A partir de estos primeros estudios, una gran cantidad de trabajos han analizado los factores determinantes del delito, mediante la inclusión de variables disuasorias, socio-económicas y demográficas ${ }^{5}$. No obstante, estas aportaciones han llegado a generar un intenso debate, no exento de cierta polémica, al que ha contribuído la diversidad de tipologías delictivas empleadas, técnicas econométricas (series temporales/sección cruzada) y niveles de agregación em-

\footnotetext{
${ }^{1}$ Véase, http://www.cis.es/baros/mar2528.doc.

${ }^{2}$ Véase, http://www.cis.es/baros/mar2511.doc.

${ }^{3}$ El texto completo puede ser encontrado en http://www.mir.es.

${ }^{4}$ Aunque sociólogos, criminólogos, y psicólogos han contribuído notablemente en este área de investigación.

${ }^{5}$ Recientemente algunos economistas han introducido nuevos elementos en la discusión (desigualdad de renta, la relación entre criminalidad y drogas, capital social y nivel de inmigración. Ver por ejemplo, Fajnzylber et al. (2002), Entorf y Winker (2001), Dilulio (1996), y Butcher and Piehl (1998).
}

Revista Española de Investigación Criminológica REIC AC 01-03 http:www.criminologia.net/revista 
pleados. En líneas generales, la mayor parte de la evidencia empírica proporciona mayor apoyo a las variables disuasorias y de caracter demográfico frente a las variables socio-económicas ${ }^{6}$

A pesar de que la literatura internacional empírica ${ }^{7}$ que se ha ocupado de este tema es amplia, en España los estudios empíricos no han sido numerosos ${ }^{8}$. El estudio más relevante para este trabajo es Bandrés y Díez-Ticio (2001), quienes estiman un modelo de ecuaciones simultáneas para analizar los determinantes de la actividad delictiva con datos del Cuerpo Nacional de Policía (CNP) de 1995. Sin embargo, desde mi punto de vista, este último trabajo no tiene en cuenta dos aspectos: (1) la existencia de algunos efectos que el investigador no puede observar, los cuales pueden tener una gran influencia sobre las tasas de delitos registrados (preferencias por el delito, diferentes grados de denuncia, etc) y (2) el ámbito temporal del conjunto de datos. Por tanto, sus resultados deberían interpretarse con cierta cautela debido a que las estimadores obtenidos podrían ser sesgados e inconsistentes en presencia de heterogeneidad inobservable. Por tanto, una metodología de panel es más adecuada ya que permite combinar información de corte transversal de $N$ individuos (comunidades autónomas) con series temporales de cada uno de ellos, y controla por la posible presencia de heterogeneidad inobservable ${ }^{9}$.

Este trabajo, por lo que a mi conocimiento respecta, es el primero que utiliza datos de panel para estudiar los factores determinantes del delito en nuestro país. La información sobre delincuencia ha sido obtenida de las estadísticas oficiales elaboradas por el Ministerio del Interior desde 1994 al año 2001.

El resto del trabajo se organiza de la siguiente forma. En la sección 2 se presenta una descripción del fenómeno delictivo. En la sección 3 se describe la muestra utilizada y la especificación econométrica. Los resultados empíricos obtenidos se presentan en la sección 4. Por último, se establecen algunas conclusiones y futuras

\footnotetext{
${ }^{6}$ Para una discusión mas detallada de los resultados empíricos obtenidos en la literatura económica ver Eide (2000).

${ }^{7}$ Dentro del ámbito europeo, algunos de los trabajos más relevantes en este campo son los de Marselli and Vannini (1997) en Italia, Sandelin and Skogh (1986) en Suecia, Entorf and Spengler (2000) en Alemania, Whalroos (1981) en Finlandia, Aasness, Eide y Skjerpen (1994) en Noruega, Wolpin (1978) en Inglaterra y Gales, Carr-Hill y Stern (1973) en Inglaterra.

${ }^{8}$ Aunque la literatura teórica ha recibido mayor atención. Véase, Montero y Torres (1998); Pastor (1993, 1996).

${ }^{9}$ Véase Baltagi (2001).
}

Revista Española de Investigación Criminológica

REIC AC 01-03 http:www.criminologia.net/revista 
extensiones del trabajo.

\section{La Actividad Delictiva en Espana}

En esta sección se presenta una breve descripción de la actividad delictiva en España con el fin de motivar el análisis empírico posterior. Todos los datos presentados en esta sección se refieren exclusivamente a los hechos delictivos conocidos por el Cuerpo Nacional de Policía (CNP) y la Guardia Civil (GC). La información sobre delincuencia procede de los Anuarios Estadísticos del Ministerio del Interior desde el año 1994 (primer año disponible) hasta el año 2001 (último año disponible).

En el cuadro 1, se presenta la evolución del delito conocido (excluyendo las faltas), el índice de criminalidad por cada 1.000 habitantes, y el porcentaje de variacion respecto al año anterior ${ }^{10}$. Como se puede observar en el cuadro 1 , la tasa de delitos registrados disminuyó entre el año 1997 y el año 2000, aunque el año 2001, ha supuesto un ligero repunte en los niveles de delincuencia, regitrándose un total de casi 24 delitos por cada 1.000 habitantes. En concreto, en el año 2001, los delitos registrados crecieron un $10 \%$ con respecto al año anterior. Durante el periodo objeto de nuestro estudio, la tasa de delincuencia aumentó en un $6 \%$. No obstante conviene matizar la información que se presenta en el Cuadro 1. Primero, consideramos que es pronto para valorar si estamos asistiendo al inicio de un aumento en los niveles de criminalidad. Segundo, un análisis temporal más amplio nos podría mostrar una mayor variabilidad en los niveles de delincuencia registrados.

\footnotetext{
${ }^{10}$ Para comparar adecuadamente se han realizado ciertos ajustes, por ejemplo, no se han incluído los delitos registrados por ambas fuerzas de seguridad del Estado en Ceuta, Melilla y el País Vasco.
}

Revista Española de Investigación Criminológica

REIC AC 01-03 http:www.criminologia.net/revista 
Cuadro 1: Delitos conocidos (delitos por cada 1.000 habitantes). CNP + GC

\begin{tabular}{lccccc}
\hline Año & $\begin{array}{c}\text { Delitos } \\
\text { conocidos }\end{array}$ & $\begin{array}{c}\text { Población } \\
\text { Total }\end{array}$ & \multicolumn{3}{c}{$\begin{array}{c}\text { Indice de delincuencia } \\
\text { (por cada 1.000 habitantes) }\end{array}$} \\
\cline { 4 - 6 } & & & \multicolumn{3}{c}{$1994=100$} \\
\hline 1994 & 887.555 & 40.229 .598 & 22.06 & 100 & $\Delta \%$ \\
1995 & 899.068 & 40.460 .055 & 22.22 & 100.73 & 0.73 \\
1996 & 922.568 & 39.669 .394 & 23.26 & 105.43 & 4.67 \\
1997 & 915.442 & 39.348 .050 & 23.24 & 105.34 & -0.09 \\
1998 & 908.565 & 39.852 .651 & 22.80 & 103.35 & -1.89 \\
1999 & 908.346 & 40.202 .160 & 22.59 & 102.40 & -0.92 \\
2000 & 872.892 & 40.499 .790 & 21.55 & 97.69 & -4.6 \\
2001 & 963.094 & 41.116 .842 & 23.42 & 106.16 & 8.67 \\
\hline
\end{tabular}

Fuentes: Anuario Estadístico del Ministerio del Interior. Ministerio del Interior. Datos oficiales de población: Instituto Nacional de Estadística (INE). Proyecciones de población a partir del censo de 1991 para el año 1997 a 1 de Julio.

También podría resultar interesante comparar la evolución temporal de los niveles de criminalidad en España con la tendencia observada en otros países de nuestro entorno, de cara a determinar si la tendencia en España es igual o difiere. El cuadro 2 muestra la evolución de la tasa general de delincuencia ${ }^{11}$ para el periodo 1994-2000 en Europa ${ }^{12}$. La última columna refleja las tasas de variacion en la tasa de inseguridad ciudadana para cada país en relación al año 1994.

En este cuadro se observa que España está muy por debajo de los niveles de delicuencia europeos. Durante el periodo 1994-2000, España registró un ligero incremento en la tasa de delincuencia (véase cuadro 2). Por el contrario, otros países europeos, como por ejemplo, Finlandia, Dinamarca, Irlanda, experimentaron descensos en el nivel de delincuencia con respecto al año 1994.

No obstante, difícilmente se pueden extraer conclusiones definitivas sobre los niveles de delincuencia que un país u otro tienen. Hay que tener en cuenta que

${ }^{11}$ Véase para más detalle Barclay y Tavares (2000, 2002).

${ }^{12}$ No existe información posterior al año 2000 
los niveles de delincuencia registrados no son homogéneos debido a que las leyes penales y procedimientos estadísticos de registro son diferentes en cada unos de los países ${ }^{13}$.

\footnotetext{
${ }^{13}$ Una notable excepción es la tasa de homicidios donde los sesgos son menores en relación a otro tipo de figuras delictivas.
}

Revista Española de Investigación Criminológica

REIC AC 01-03 http:www.criminologia.net/revista 


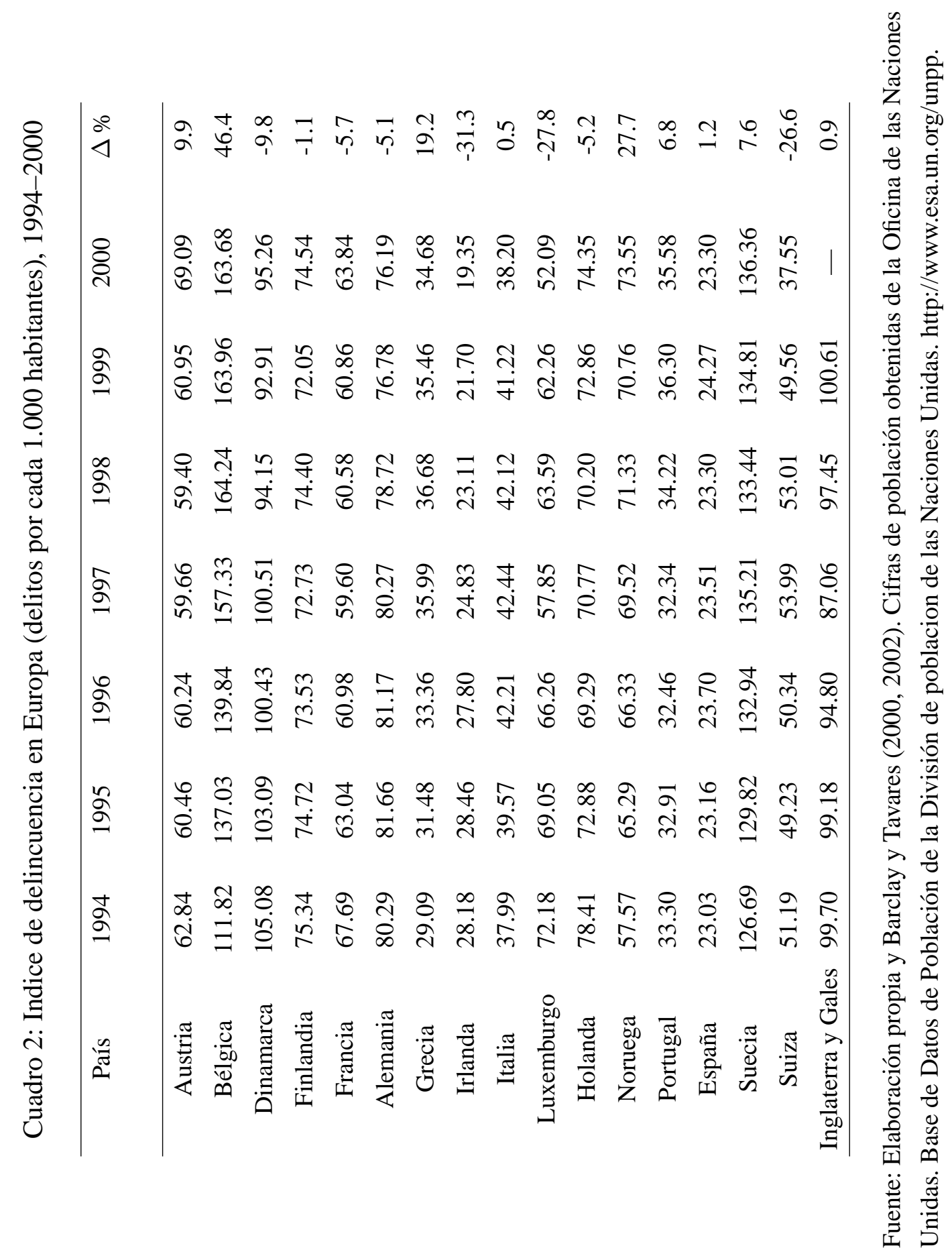

Revista Española de Investigación Criminológica REIC AC 01-03 http:www.criminologia.net/revista 
Una vez presentados los índices generales de criminalidad en Europa, pasaremos a estudiar la tipología delictiva en España. En el cuadro 3, se muestran las principales figuras delictivas conocidas por el CNP y la GC y su proporción con respecto al total de delitos conocidos en el año respectivo. La última columna refleja las variaciones de los delitos contra el patrimonio, libertad sexual y contra las personas en relación al año 1994. Prácticamente el $86 \%$ de los delitos registrados son delitos contra el patrimonio y orden socio-económico. Los delitos contra las personas y libertad sexual representan aproximadamente un $2 \%$ y un $1 \%$ del total de delitos conocidos respectivamente. En relación a la evolución temporal de los diferentes delitos, observamos un crecimiento generalizado, si bien los delitos contra las personas parecen mostrar una mayor variación. Nótese que la distribución de la tipologia delictiva permanece casi inalterada durante el periodo de análisis.

Cuadro 3: Tipos de delitos. CNP+GC

\begin{tabular}{cccccccc}
\hline Tipo de delito & \multicolumn{2}{c}{1994} & & \multicolumn{2}{c}{2001} & & $\Delta \%$ \\
\cline { 2 - 3 } & Delitos & \% total de & & Delitos & $\%$ total de & & \\
& conocidos & delitos & & conocidos & delitos & \\
\hline contra las personas & 14.651 & 1.65 & & 16.618 & 1.73 & & 13.42 \\
contra la propiedad & 762.260 & 85.88 & & 845.512 & 87.79 & & 10.92 \\
contra la libertad sexual & 6.233 & 0.70 & & 6.824 & 0.71 & & 9.48 \\
\hline Total de delitos & 887.555 & & & 963.094 & & \\
conocidos & & & & & \\
\hline
\end{tabular}

Fuente: Elaboración propia y Anuarios Estadísticos del Ministerio del Interior.

El cuadro 4 permite comparar la evolución temporal de la tasa de delitos registrados por comunidades autónomas. La última columna expresa las variaciones en la tasa de delicuencia por provincias en relación al año 1994. En el se aprecia las grandes diferencias en los niveles de delincuencia a nivel autonómico. Unas 
provincias registran mayores tasas de delincuencia que otras. Por ejemplo, Baleares registró la tasa más alta de delicuencia con 47.7 delitos por cada 1.000 habitantes en el 2001, seguida por Canarias con 34.6 delitos por 1.000 habitantes. Las tasas más bajas de delitos se encuentran en Castilla-León, y Castilla La Mancha con 11 y 13 delitos por cada 1.000 habitantes respectivamente. Por otra parte, destaca el crecimiento en la tasa de delitos registrados en el periodo 1994-2001, salvo Asturias, Madrid, Cataluña, Canarias. Cantabria, Castilla-León, y Galicia.

En el Cuadro 5, se muestra la evolución de la tasa de delitos esclarecidos por comunidades autonomas. En la última columna se muestran las tasas de variación de la tasa de delitos esclarecidos también por comunidades autónomas. La tasa de delitos esclarecidos experimentó un crecimiento positivo a lo largo del periodo 1994-2001 del 4.5\%. Las diferencias en las tasas de delitos esclarecidos a nivel provincial son evidentes. Por ejemplo, en el 2001, Cantabria (47.25) y Asturias (44.56) registraron las tasas de delitos esclarecidos más altas en comparación con Madrid (18.02), Valencia (21.67) y Aragón (21.58). Por otra parte, notable es el descenso experimentado en la tasa de delitos esclarecidos en Aragón y Cataluña con un 37 y 19 por ciento respectivamente.

En cuanto a los aspectos socio-demográficos del crimen, existe la percepción de que en los ultimos años ha habido un incremento en el número de immigrantes detenidos. Sin embargo, algunas cuestiones deben ser matizadas al respecto. En primer lugar, es importante diferenciar entre los extranjeros detenidos por motivos administrativos (estancia ilegal) o penales ${ }^{14}$. Un hecho constatado es que la mayoría de los extranjeros detenidos son detenidos debido a su situación irregular y no por motivos penales. El cuadro 6 presenta la cifra de detenciones de españoles y extranjeros efectuadas por el CNP y la GC por motivo de delito, falta y reclamación judicial. La última fila son las tasas de variaciones con respecto al año 1994. Aún estando presente un cierto margen de error debido a la falta de información respecto a las detenciones efectuadas por las fuerzas policiales autonómicas, el cuadro 6 revela que a partir de 1997 la cifra de detenciones de ciudadanos españoles se ha reducido, mientras que ha habido un aumento con-

\footnotetext{
${ }^{14}$ Sólo a partir de 1997, las estadísticas oficiales del Ministerio del Interior distinguen entre detenciones por motivos administrativos y penales.
}

Revista Española de Investigación Criminológica

REIC AC 01-03 http:www.criminologia.net/revista 


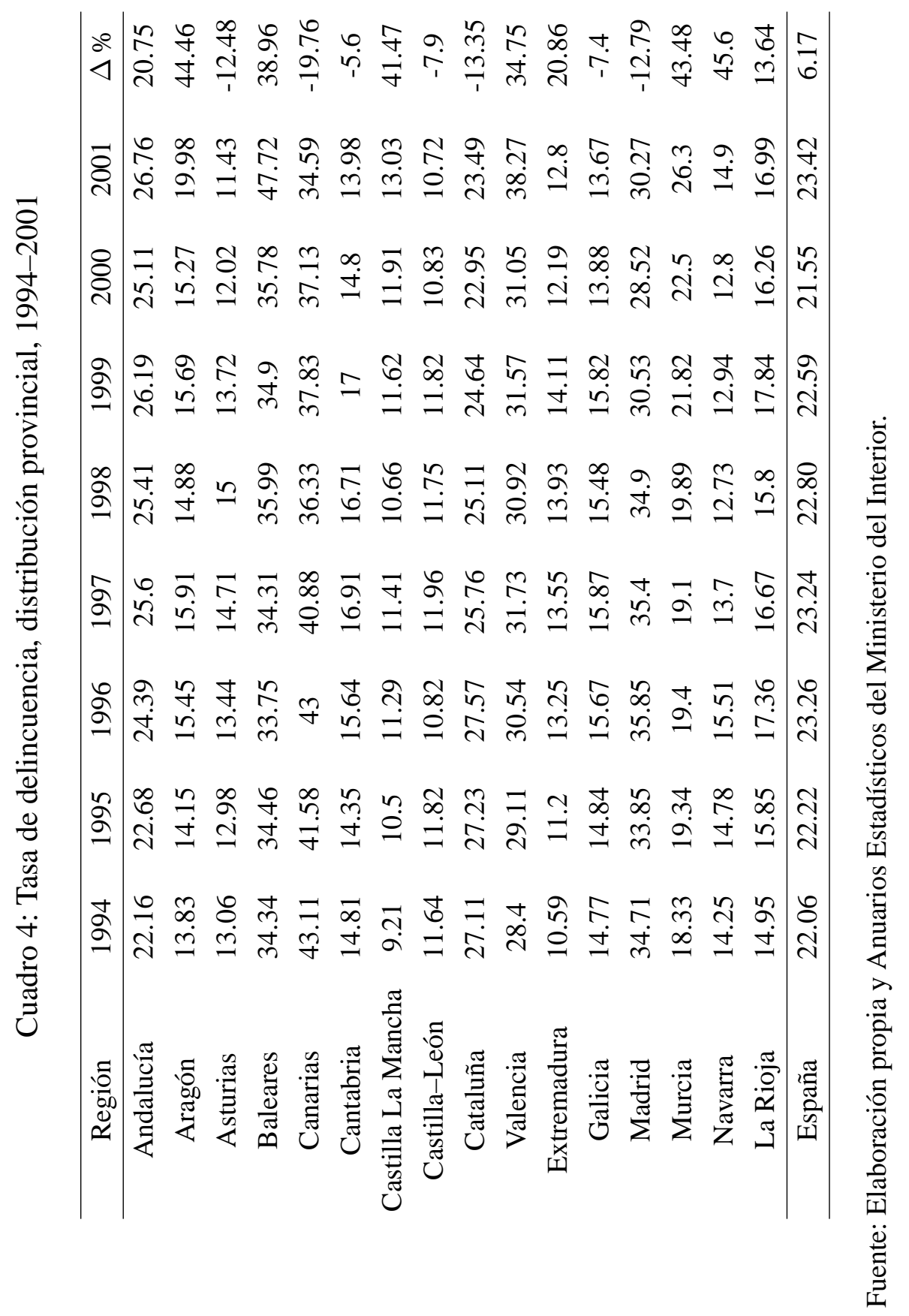

Revista Española de Investigación Criminológica REIC AC 01-03 http:www.criminologia.net/revista 


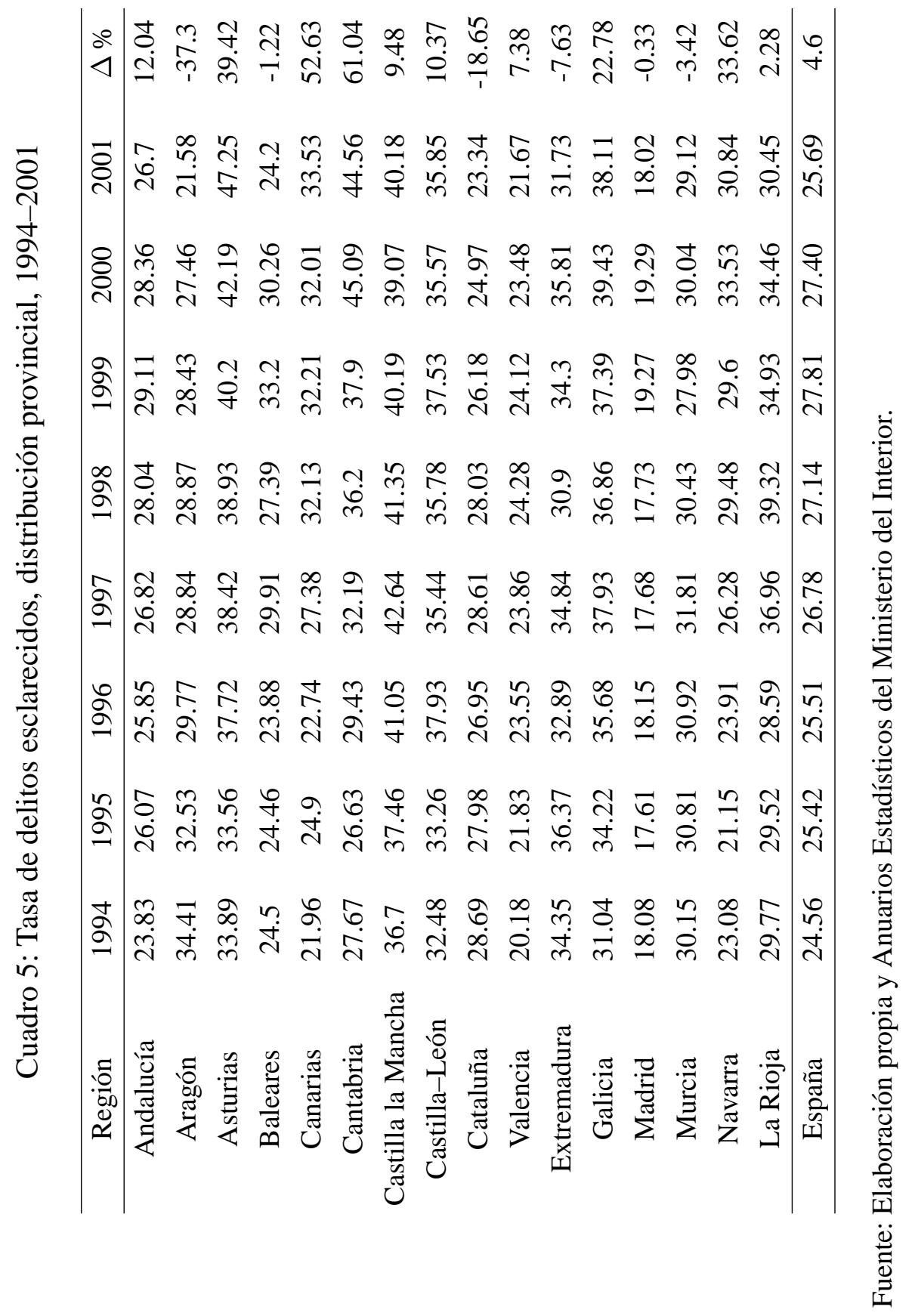

Revista Española de Investigación Criminológica REIC AC 01-03 http:www.criminologia.net/revista 
siderable en la cifra de detenidos extranjeros. En particular, durante el periodo 1994-2000, el número de extranjeros detenidos ha aumentado un $97 \%$ con respecto a 1994 mientras que el aumento en el número de españoles detenidos ha sido de sólo un 12 por ciento.

Cuadro 6: Detenciones por infracciones penales (delitos, faltas y reclamaciones judiciales). CNP+GC. 1994-2000.

\begin{tabular}{cccccc}
\hline Año & Españoles & Extranjeros & $\begin{array}{c}\text { Total } \\
\text { detenidos }\end{array}$ & $\begin{array}{c}\text { Población } \\
\text { española }\end{array}$ & $\begin{array}{c}\text { Población } \\
\text { Extranjera }\end{array}$ \\
\hline 1994 & 219.629 & 28.250 & 247.879 & 40.229 .598 & 461.364 \\
1995 & 215.448 & 29.718 & 245.166 & $40,460,055$ & 499.773 \\
1996 & 222.095 & 32.001 & 254.096 & 39.669 .394 & 538.984 \\
1997 & 211.028 & 35.991 & 247.199 & $39.348,050$ & 609.813 \\
1998 & 204.436 & 37.617 & 242,053 & 39.852 .651 & 719.647 \\
1999 & 205,002 & 41.887 & 246.889 & 40.202 .160 & 801.324 \\
2000 & 196.459 & 55.693 & 253.625 & 40.499 .790 & 895.720 \\
\hline$\Delta \%$ & -12 & 97 & 2.3 & 0.67 & 94.15 \\
\hline
\end{tabular}

Fuente: Elaboración propia y Anuarios Estadísticos del Ministerio del Interior. Datos oficiales de población: Instituto Nacional de Estadística.

Sin embargo, hay que matizar que no todos los extranjeros detenidos por la policía son residentes legales. De acuerdo a un estudio realizado en las cárceles españolas, sólo el $78 \%$ de los presos eran residentes legales (García, 2001). Además estas cifras deberían ponerse en relación con los correspondientes grupos de población (española y extranjera) para tener una percepción adecuada de la relación entre inmigración y delincuencia. Algunos estudios para el caso español han concluído que es mayor la tasa de delincuencia de la población extranjera que la de la población autóctona (García y Prieto, 1997). Mientras que otros autores afirman que estas cifras podrían ser reflejo de prácticas policiales que discriminan a los extranjeros. A este respecto, estudios realizados en diversos países europeos restan credibilidad a dicho argumento (Killias, 2001).

Para finalizar, resulta interesante comprobar, que los varones son más propen-

Revista Española de Investigación Criminológica REIC AC 01-03 http:www.criminologia.net/revista 
sos que las mujeres a cometer delitos. En el año 2001, un total de 26.504 menores de 18 años fueron detenidos por los cuerpos de seguridad del estado, representando un aumento de casi el 82 por ciento con respecto al año anterior. Aproximadamente, un 92 por ciento fueron hombres mientras que solamente el 8 por ciento fueron mujeres. El tipo de delito mayormente cometido por la población joven es contra el patrimonio representando un 75 por ciento del total de delitos.

\section{Datos}

Los datos sobre delincuencia utilizados en este trabajo han sido extraídos de los Anuarios Estadistícos del Ministerio del Interior ${ }^{15}$. Estos recogen información sobre la delincuencia registrada por las Fuerzas y Cuerpos de Seguridad del Estado en todo el territorio nacional. Algunas consideraciones sobre los datos son de interés. Para evitar la existencia de ciertos problemas de comparabilidad que surgen por la coexistencia de policias autonómicas con las fuerzas estatales, nos centraremos en los delitos registrados por las dos principales fuerzas de Seguridad del Estado: el CNP y la GC. Algunas otras consideraciones sobre la muestra utilizada son de interés. Ceuta y Melilla fueron excluídas del análisis tanto por sus peculiaridades como por su reducida dimensión. Se ha excluído también el País Vasco debido a la alta incidencia de actividades terroristas y a la falta de homogeneización de la información con el Programa Estadístico operado por el Ministerio del Interior.

La muestra final está formada por 16 comunidades autónomas para el periodo 1994-2001. La elección del periodo muestral viene condicionada por la información existente sobre sobre delincuencia, dicha información no es disponible con anterioridad al año 1994. (Line from his log files.) The effect is that the definition

\footnotetext{
${ }^{15}$ Una fuente alternativa a los datos oficiales sobre delincuencia son las encuestas de victimización. Esta técnica consiste en preguntar a una muestra representativa de la población si han sido objeto de algún tipo de delito durante el ultimo año. El Centro de Investigaciones Sociológicas (CIS) ha llevado a cabo encuestas nacionales en 1978, 1991, 1992, 1995,1996 y 1999. Sin embargo, una gran limitación es que los delitos no son comparables entre las distintas encuestas. Para una discusión sobre los problemas del uso de las Encuestas de Victimizacion, véase, Stageland (2001).
}

Revista Española de Investigación Criminológica REIC AC 01-03 http:www.criminologia.net/revista 


\subsection{Modelo empírico}

A partir del modelo téorico, inspirado en Becker (1968) y Ehrlich (1973), se propone un modelo econométrico de datos de panel con el objeto de contrastar una serie de hipótesis en la literatura teórica y empírica sobre los determinantes de la actividad delictiva ${ }^{16}$.

El modelo teórico asume que los individuos son maximizadores de la utilidad, tienen información perfecta y son neutrales al riesgo. Bajo estas condiciones, los individuos elegirán cometer un delito si los beneficios esperados de las actividades ilegales son superiores a los costes (véase, Becker 1968; Ehrlich, 1973; Block and Heineke, 1975; and Schmidt and Witte, 1984). Obviamente, lo ideal sería de disponer de datos individuales, pero esto no es posible dado que la información oficial sobre las actividades delictivas es disponible a nivel agregado ${ }^{17}$. La especificación del modelo de regresión que vamos a utilizar para analizar los determinantes socio-económicos del delito viene dada por la ecuación:

$$
Y_{i t}=\alpha_{i}+\gamma_{t}+X_{i t}^{\prime} \beta+\epsilon_{i t} \quad i=1, \ldots, N . \quad t=1, \ldots, T .
$$

donde $Y_{i t}$ es la variable dependiente. El término $\alpha_{i}$ representa el efecto individual específico de la provincia $i$, que se supone le afecta por igual a lo largo del tiempo (por ejemplo, actitud hacia el delito, diferentes grados de denuncia del delito), el término $\gamma_{t}$ representa el efecto temporal específico para el periodo $t$ que se supone afecta por igual a todas las regiones (por ejemplo, cambios en el codigo penal, políticas macroeconómicas, etc). $X_{i t}$ es un vector que contiene tres tipos de variables explicativas: disuasorias, socio-económicas y demográficas, y $\epsilon_{i t}$ es un término de error que se supone independiente e idénticamente distribuido con media cero y varianza constante. En la terminología de datos de panel, es simplemente un modelo de efectos fijos ${ }^{18}$. Nótese que no hacemos ningun supuesto a priori sobre la posible correlación entre los efectos individuales y el término de

\footnotetext{
${ }^{16}$ Aunque existen otras diversas teorías acerca del crimen, por ejemplo, la teoría del control social (Hirschi 1969), la teoría del estilo de vida (Cohen y Felson, 1979) y la teoría del strain (Cohen, 1955).

${ }^{17} \mathrm{El}$ primer estudio contrastando la teoría formulada por Becker utilizando datos individuales fue Witte (1980).

${ }^{18}$ Alternativamente, podríamos considerar $\operatorname{los} \alpha_{i}$ como variables aleatorias, en lugar de como parametros a estimar, entonces tendríamos el modelo de efectos aleatorios.
} 
error.

\section{Las variables}

A continuacion se presentan las variables que permitirán contrastar empíricamente las hipótesis planteadas en el modelo económico del crimen.

\section{- La variable dependiente}

Nuestra variable dependiente es la tasa de delitos registrados (TDREG), la cual se define como el cociente entre el número total de delitos conocidos por el CNP y la GC y la población total en cada comunidad autónoma $i$ en el periodo $t$. Como no es posible cuantificar la delincuencia real, debido a que no todos los delitos son dados a conocer a las fuerzas policiales, esta variable es la mejor aproximación que el investigador dispone para cuantificar la delincuencia. En nuestra variable, $T D R E G$, incluímos todos los delitos tipificados en el Codigo Penal y leyes penales especiales, excluyendo las faltas.

Dado que no todos los delitos obedecen a los mismos motivos (una violación no es igual que un atraco o un delito monetario), y que no todos lo delitos son registrados de igual manera, esto es de alguna manera cuestionable. No obstante, la razón de elegir una variable agregada fue a que no se dispone de información con respecto a las variables disuasorias por tipo de delito durante el periodo de estudio, lo que reduce el tamaño muestral del estudio a 90 observaciones. Por otro lado, al considerar la tasa agregada de delincuencia, y dado que el delito más común es aquel contra la propiedad, la variación en la variable TDREG está dominada por las fluctuaciones en la serie temporal de los delitos contra el patrimonio.

\section{- Las variables explicativas}

Probabilidad de captura (TDESC). Las variables disuasorias pretenden capturar los efectos de cambios en la probabilidad de castigo sobre las actividades criminales ${ }^{19}$. En general se espera que cuanto mayor sea la probabilidad percibida

${ }^{19}$ Véase Nagin (1998) para una excelente revisión sobre el empleo de variables disuasorias en modelos empíricos. 
de captura mayor será el coste de oportunidad de las actividades ilegales reduciendo así la participación de los individuos en actividades ilegales (Becker, 1968). La proxy variable que más se ha utilizado en la literatura para medir la probabilidad de captura es la tasa de delitos esclarecidos la cual se define como el cociente entre el número de delitos resueltos sobre el total de delitos registrados. La variable TDESC ha sido obtenida de los Anuarios Estadísticos del Ministerio del Interior.

También se podría aproximar la probabilidad de captura mediante el número de efectivos policiales (CNP+ GC), en cada comunidad autónoma, sin embargo, no existe información completa de esta variable en el periodo de estudio para ambas fuerzas policiales ${ }^{20}$. Por otro lado, no hemos incluído en el análisis una variable que permita aproximar la severidad de las penas, por ejemplo, la duración media de las sentencias condenatorias. Sin embargo, creemos que este efecto podría ser capturado a través de los efectos específicos individuales, suponiendo que la variación en la severidad de las penas es pequeña lo largo del tiempo y difiere en cada provincia.

Tasa de desempleo (PARO). La inclusión de esta variable está motivada por el hecho ampliamente constatado en estudios empíricos, de que refleja la falta de oportunidades de conseguir una renta procedente de actividades legales ${ }^{21}$. Si las oportunidades de obtener una renta de actividades legales son relativamente escasas en relación a los beneficios potenciales que se derivan del delito, la teoría económica predice que los individuos serán más propensos a delinquir. El desempleo refleja el coste de oportunidad de las actividades ilegales haciendo el delito más atractivo. Otra parte de la literatura (Cantor y Land, 1985; Cook and Harkin, 1985; Smith, Devine y Sheley, 1992; y Greenberg, 2001) argumenta que existe un efecto oportunidad. Los desempleados podrían pasar más tiempo en casa, impidiendo cierto tipo de delitos (homicidios, robos, etc). La tasa de desempleo ha sido recogida de las Estadísticas Anuales de la Encuesta de Población Activa (EPA) del Instituto Nacional de Estadística (INE).

\footnotetext{
${ }^{20}$ Sólo se dispone del año 2000.

${ }^{21}$ Véase Chiricos (1987) y Freeman (1983) para una exhaustiva revisión de la evidencia empírica entre el nivel de desempleo y los niveles de delincuencia.
}

Revista Española de Investigación Criminológica REIC AC 01-03 http:www.criminologia.net/revista 
PIB per capita (RENTA). Las oportunidades de obtener una renta procedente de actividades legales son aproximadas mediante la renta per capita (Trumbull, 1989). Cuanto mejor sean las condiciones económicas es más probable que los individuos puedan obtener unos beneficios procedentes de actividades legales y por tanto se registren unos niveles de delincuencia menores. Por otra parte, en la literatura, se ha ofrecido otra explicación alternativa. Los beneficios potenciales de actividades ilegales son mayores en regiones prósperas, en la medida en que las posibilidades de obtener un botín más suculento son mayores. En este sentido cabría esperar que el signo del parámetro asociado a esta variable sea positivo (Ehrlich (1973); Avio y Clark (1978); Sala-i-Martín (1997) y Entorf y Spengler (2000).). La variable RENTA procede de las series anuales de Contabilidad Nacional elaboradas por el Instituto Nacional de Estadística (INE) y está expresado en miles de pesetas constantes del año 1994.

Nivel de estudios (EDUC). La relación entre la actividad delictiva y el nivel de estudios ha sido apoyada por diversos trabajos empíricos (Ehrlich, 1975; Lochner, 2000). El nivel educativo de los individuos constituye en gran medida un indicador de la posible generación de ingresos procedentes de actividades legales. De esta manera, unos mayores niveles de educación estarían asociados con unos mayores ingresos futuros y por tanto con unos costes de oportunidad del delito mayores. Atendiendo a esto motivos, cabría esperar un signo negativo en la relación entre delincuencia y educación. La variable EDU es la proporción de la población activa con estudios primarios y ha sido obtenida de Mas et al. (2002).

Inmigrantes extranjeros (INMIGRA). Siguiendo estudios recientes (Entorf y Spengler, 2000; Entorf y Winker, 2001), hemos tenido en cuenta como variable explicativa los niveles de inmigracion. Cabe pensar que los inmmigrantes irregulares (sin un permiso legal de residencia) pueden tener una relación más estrecha con los niveles de criminalidad, al disponer de menos oportunidades de actuar dentro de un marco legal y terminen delinquiendo para sobrevivir. En este sentido la teoría del arraigo social formulada por el criminólogo Hirschi (1969) proporciona una respuesta en cuanto a la dirección del efecto de la inmigración sobre los niveles de delincuencia. De acuerdo a esta teoría, el proceso de la inmigración 
supone una ruptura con los lazos familiares pudiendo llevar a una pérdida de autoestima, reconocimiento social y por tanto siendo los inmigrantes más propensos a delinquir que aquellas personas que disponen de tales vinculos sociales. Tampoco ha sido posible incluír ciertas características individuales de los inmigrantes como el nivel de renta y educativo que podrían tener un papel importante a la hora de analizar las causas de por qué los inmigrantes delinquen más.

Con el fin de determinar si existe una relación de causalidad entre inmigración y delincuencia, la informacion más adecuada sería el numero de inmigrantes ilegales. Obviamente, no se dispone de información estadística oficial fiable sobre el número de inmigrantes en situación irregular. A falta de dicha información, una manera de evaluar el impacto de la inmigración sobre la delincuencia, es utilizar como aproximación el número de residentes legales extranjeros. La variable $I N M I G R A$ es la proporción de residentes legales extranjeros y ha sido obtenida del Instituto Nacional de Estadística (INE).

Jóvenes (JOV). Para el estudio de la delincuencia, resulta fundamental incluír una variable controlando por la edad de los potenciales delincuentes (Marvell y Moody, 1991). Como se comprobó anteriormente, el colectivo entre 16 y 24 años, especialmente hombres, es el que tiene una mayor propensión a delinquir. Esto podría ser debido a la falta de oportunidades de actuar dentro de la legalidad, a un bajo poder adquisitivo, ó a un intento de suscitar la atención dentro del ambito familiar. La variable $J O V$ representa la proporción de hombres entre 16 y 24 años y ha sido obtenida del Instituto Nacional de Estadística.

Densidad de población (DEN). En general se espera que la conductas delictivas sean mayores en zonas con una mayor densidad de población. Por ejemplo, en áreas con una alta densidad de población, la probabilidad de ser capturado es menor, induciendo a los individuos a cometer más delitos. La variable $D E N$ es medida como el número de habitantes por $K \mathrm{~m}^{2}$ y ha sido obtenida del Instituto Nacional de Estadística.

En el cuadro 7 se muestran los principales estadísticos descriptivos de todas las variables utilizadas en el análisis empírico.

Revista Española de Investigación Criminológica REIC AC 01-03 http:www.criminologia.net/revista 
Cuadro 7: Estadísticas descriptivas (Tamaño muestral=128)

\begin{tabular}{cccccc}
\hline Variables & Media & Desv. Típica & Mínimo & Máximo & $S / X$ \\
& $(\mathrm{X})$ & $(\mathrm{S})$ & & & \\
& & & & & \\
\hline TDREG & 21.03 & 9.41 & 9.21 & 47.72 & 0.45 \\
RENTA & 1,944 & 410.58 & 1,139 & 2,915 & 0.21 \\
TDESC & 30.58 & 6.55 & 17.61 & 47.25 & 0.21 \\
PARO & 17.41 & 6.41 & 5.88 & 34.63 & 0.37 \\
DEN & 129.55 & 146.32 & 21.33 & 669.21 & 1.13 \\
INMIGRA & 1.63 & 1.26 & 0.32 & 5.42 & 0.77 \\
JOV & 7.06 & 0.59 & 5.42 & 8.33 & 0.08 \\
EDUC & 32.64 & 6.91 & 18.22 & 51.58 & 0.21 \\
\hline
\end{tabular}

\section{Resultados}

En el cuadro 9 se presentan las estimaciones del modelo de efectos fijos, y en la última colunma se presentan las correspondientes al resultado de aplicar el metodo de variables instrumentales al modelo de efectos fijos. Siguiendo anteriores estudios empíricos, todas las variables se especifican en logaritmos. Esta especificación es particularmente útil, porque los coeficientes obtenidos pueden ser directamente interpretados como elasticidades.

Los resultados del cuadro 8 indican que la heterogeneidad inobservable capturada por las variables ficticias individuales y temporales debe ser teniada en cuenta en el análisis, y apoya, por tanto, la especificación inicial de nuestro modelo empírico ${ }^{22}$.

A continuación se examina si los efectos individuales están correlacionados con las variables explicativas mediante el contraste propuesto por Hausman (1978). Bajo la hipótesis nula de no correlación entre los efectos individuales y las variables explicativas el estadístico se distribuye como una chi-cuadrado con tantos grados de libertad como el número de variables explicativas, excluyendo el término constante. En este caso, el contraste de Hausman rechaza la hipótesis nula de

${ }^{22}$ Véase Baltagi (2001), para una descripción más detallada de los estadísticos. 
ausencia de correlación entre los efectos individuales y las variables explicativas a un nivel de significación del $95 \%$ (p-valor= 0.002), y por tanto, el modelo de efectos fijos (WITHIN) es preferible, ya que es el único estimador consistente ${ }^{23}$.

Cuadro 8: Contrastes de Heterogeneidad

\begin{tabular}{ccccc}
\hline $\begin{array}{c}\text { Hipótesis } \\
\text { nula }\end{array}$ & F-estadístico & GL(N) & GL(D) & p-valor \\
\hline$H_{0}: \alpha_{i} \neq 0, \gamma_{t}=0$ & 19.77 & 15 & 105 & 0.0001 \\
$H_{0}: \alpha_{i}=0, \gamma_{t} \neq 0$ & 3.14 & 7 & 113 & 0.0046 \\
$H_{0}: \alpha_{i}=0, \gamma_{t}=0$ & 19.55 & 22 & 98 & 0.0001 \\
\hline
\end{tabular}

${ }^{\text {a }}$ GL(N): Grados de libertad (numerador).

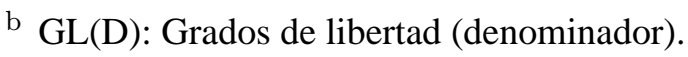

En primer lugar, en el cuadro 9 se observa que todas las variables son significativas a los niveles convencionales de significación y con los signos esperados. Sin embargo, en este contexto parece razonable plantearse que la variable TDESC no sea totalmente exógena, en el sentido de que, por ejemplo, la tasa de delitos esclarecidos es determinada por el nivel de delincuencia, y por tanto estaría correlacionada con el término error, invalidando la consistencia del estimador intragrupos.

Para contrastar la posible endogeneidad de la variable $T D E S C$ se ha seguido un procedimiento en dos etapas. En la primera etapa, se estima un modelo donde la variable potencialmente endógena es relacionada al resto de variables explicativas y el conjunto de instrumentos. En la segunda etapa, los residuos obtenidos en la estimación anterior son incluídas como un regresor adicional en la ecuación estructural y si el coeficiente correspondiente es significativo, indica que la probabilidad de captura es endógena. Tras aplicar el contraste, no podemos aceptar la hipótesis nula de exogeneidad de la variable TDESC (p-valor=0.0002), lo que indica que existe un problema de endogeneidad en nuestro modelo. Por tanto, la estimación intra-grupos (WITHIN) será inconsistente en presencia de endogenei-

\footnotetext{
${ }^{23}$ Obviamente, si los efectos individuales no estuvieran correlacionados con las variables explicativas, el modelo de efectos aleatorios hubiera sido una mejor alternativa, ya que no sólo es consistente sino más eficiente que el estimador intra-grupos (WHITIN).
}

Revista Española de Investigación Criminológica

REIC AC 01-03 http:www.criminologia.net/revista 
dad de las variables explicativas.

Así se ha llevado a cabo la estimación del modelo de efectos fijos por el método de variables instrumentales (VI). Para ello debemos instrumentar la posible variable endógena, en este caso, la probabilidad de captura, teniendo en cuenta que los instrumentos utilizados deben estar correlacionados con la variable potencialmente endógena y no con el término de error. Para ello hemos elegido como instrumento la propia variable retardada un periodo, al no disponer de instrumentos externos adecuados. En este sentido conviene señalar que ha habido un gran esfuerzo en la literatura económica por encontrar instrumentos adecuados para resolver el problema de simultaneidad entre la tasa de delicuencia y las variables disuasorias ${ }^{24}$. La validez de los instrumentos es contrastada mediante un contraste de Basmann (1960). El test de Basmann acepta la hipótesis nula de validez de los instrumentos a niveles convencionales de significación ${ }^{25}$.

Por último, pasamos a discutir los resultados de la estimación del modelo de efectos fijos por variables instrumentales (VI). Observando la columna segunda del cuadro 9, vemos que la mayor parte de los parámetros son significativos y con el signo de acorde a lo esperado.

En lo que respecta a las variables disuasorias podemos destacar lo siguiente. En primer lugar, el coeficiente asociado a la variable TDESC es negativo pero deja de ser significativo cuando si lo era en el modelo anterior, mostrando que un aumento en la probabilidad de captura reduce los niveles de delincuencia, al incrementar el coste de oportunidad de las actividades ilegales. En concreto, el parámetro estimado disminuyó en un $50 \%$ pasando de -0.2662 a -0.1352 . En el trabajo de Cornwell y Trumbull (1994) también se encontraba esta relación negativa y no significativa, aunque ellos utilizaban como aproximación a la probabilidad de captura la tasa de arrestos.

Con respecto a las variables socio-económicas, la variable que mide la tasa de paro (PARO) muestra un signo de acuerdo a lo esperado, sin embargo, no resultó significativa. Este resultado es congruente con lo obtenido en otros estudios

\footnotetext{
${ }^{24}$ Algunos trabajos en la literatura económica que han estudiado el problema de simultaneidad son Marvell y Moody (1996); Levitt $(1996,1997)$.

${ }^{25} \mathrm{El}$ p-valor del estadístico es de 0.02 .
}

Revista Española de Investigación Criminológica

REIC AC 01-03 http:www.criminologia.net/revista 
que utilizan datos de panel (Entorf and Spengler, 2000; Small and Lewis, 1996; Papps and Winkelman, 1998). La relación inversa y significativa entre el nivel de renta y los niveles de delicuencia está en concordancia con la idea de que la renta mide los beneficios potenciales de las actividades legales.

De interés es la relación negativa y significativa del nivel de educación (EDUC). De esta manera puede argumentarse que la educación es una proxy variable de los rendimientos esperados de las actividades legales. Este resultado coincide con el obtenido en otros esdutios (Ehrlich, 1975; Trumbull, 1989 y Lochner, 2000).

Cuadro 9: Determinantes Socio-económicos del delito en España ${ }^{\mathrm{a}}$, 1994-2001.

\begin{tabular}{|c|c|c|c|c|}
\hline \multirow[b]{2}{*}{ Variable } & \multicolumn{2}{|r|}{ Efectos Fijos } & \multicolumn{2}{|c|}{ Efectos Fijos-VI } \\
\hline & coeficiente & error estándar & coeficiente & error estándar \\
\hline TDESC & $-0.2662^{*}$ & 0.0599 & -0.1352 & 0.1318 \\
\hline PARO & $0.2106^{*}$ & 0.06812 & 0.0652 & 0.0892 \\
\hline RENTA & $-0.5121^{* *}$ & 0.2331 & $-1.5518^{*}$ & 0.3897 \\
\hline$E D U C$ & $-0.2673^{*}$ & 0.0902 & $-0.3514^{*}$ & 0.1297 \\
\hline$J O V$ & $0.9773^{*}$ & 0.2330 & $1.1111^{*}$ & 0.3897 \\
\hline INMIGRA & $0.1500^{*}$ & 0.0410 & $0.1183^{* *}$ & 0.0599 \\
\hline$D E N$ & $1.5456^{*}$ & 0.3807 & & \\
\hline Num. de observaciones & 128 & & 112 & \\
\hline Sigf. Conjunta & 215.87 & & 179.62 & \\
\hline (p-valor) & $(0.0001)$ & & $(0.000)$ & \\
\hline$R^{2}$ & 0.98 & & 0.97 & \\
\hline
\end{tabular}

Por lo que respecta a las variables de corte demográfico, la variable INMIGRA, presenta un signo positivo y significativo. Sin embargo, debido al alto nivel de agregación con el que trabajamos, no es posible determinar si es debido a discriminación en el mercado de trabajo, bajos niveles educativos u otros factores. Estos

Revista Española de Investigación Criminológica REIC AC 01-03 http:www.criminologia.net/revista 
resultados contrastan con los obtenidos por Entorf y Spengler (2000), donde no se encontraba un efecto significativo. Por su parte en el trabajo de Bandrés y DíezTicio (2001), aunque se utilizó como variable, también se encontró una relación positiva y significativa.

Como era previsible, la variable $D E N$ influye positivamente en el nivel de delincuencia y de forma significativa, confirmando así el efecto esperado. Este resultado parece razonable en la medida en que en las grandes ciudades los individuos perciben un menor riesgo de ser capturados, debido al posible anonimato, y por tanto unos costes esperados de las actividades ilegales menores.

Para finalizar, el signo del estimador para la variable $J O V$ es positivo y significativo tal como en Entorf y Spengler (2000).

Para concluir, debemos resaltar que nuestro difiere del de Bandrés y DíezTicio (2001), en lo que respecta a la no significatividad de las variables disuasorias. Estas diferencias podrían estar motivadas por el uso de diferentes fuentes de datos, periodo muestral y técnicas econométricas empleadas.

Revista Española de Investigación Criminológica

REIC AC 01-03 http:www.criminologia.net/revista 


\section{Conclusiones}

Este trabajo es el primero que analiza los determinantes socio-económicos de la actividad delictiva en España utilizando técnicas de datos de panel. Los resultados son congruentes con estudios empíricos previos. Adicionalmente, se examina la posible endogeneidad de la variable empleada para aproximar la probabilidad percibida de captura. Las estimaciones del modelo de efectos fijos por variables instrumentales muestran que las variables demográficas parecen tener un mayor impacto que las variables socio-económicas para explicar variaciones en los niveles de delincuencia. Asímismo, la relación negativa entre la tasa de delitos esclarecidos y los niveles de delincuencia resultó no significativa. Los resultados también demuestran la importancia de otras variables como la educación, la renta, la proporción de hombres jovenes entre 16 y 24 años y la inmigración a la hora de explicar las variaciones en los niveles de delincuencia. Aunque en el caso de la inmigración, los resultados deben ser tomados con ciertas reservas debido a la limitación de no poder contar con la información sobre la inmigración ilegal y no poder tampoco distinguir si el efecto es debido a características individuales de los inmigrantes (por ejemplo, niveles de renta y educativos).

Este artículo pretende ser un punto de partida en el estudio empírico de la delincuencia en España. Sin embargo, algunas extensiones merecen ser consideradas. En primer lugar, los resultados podrían sufrir de un cierto sesgo por agregación (Cherry y List, 2002). Utilizar información sobre los diferentes tipos de delitos enriquecería el análisis. Los robos o hurtos, o asesinatos no son motivados por los mismos motivos, y por tanto, cabría esperar que las variables explicativas tengan un impacto diferente según el tipo de delito considerado.

En segundo lugar, sería interesante desagregar el análisis a nivel provincial. Es verdad que las provincias de una comunidad autónoma son homogéneas?. Las comunidades autónomas se componen de zonas rurales y urbanas donde las decisiones de cometer un delito son bastante heterogéneas.

Las implicaciones en términos de política económica que se derivan de este trabajo son relevantes. Este estudio sugiere que la educación ejerce una influen- 
cia positiva sobre la delincuencia, incrementando los costes de oportunidad de las actividades delictivas. Por tanto, las instituciones deberían de fomentar políticas educativas destinadas a aumentar los niveles de escolarización de la población. La renta y los niveles de empleo ejercen una influencia positiva sobre la delincuencia, y por tanto medidas de política económica que favorezcan el crecimiento económico y la mejora en los niveles de empleo desincentivarían las conductas delictivas de los individuos. Para finalizar, medidas orientadas a facilitar la integración social de los inmigrantes, haciéndoles sentir identificados con los valores del país, y por tanto verse menos expuestos a un situación de riesgo delictivo podrían tener un impacto positivo sobre la delincuencia. Por tanto, la lucha contra la delincuencia debe de ser abordada no solamente desde una perspectiva macroeconómica sino también desde una perspectiva social.

Revista Española de Investigación Criminológica REIC AC 01-03 http:www.criminologia.net/revista 


\section{Referencias}

[1] Aasness, J., Eide, E. y T. Skjerpen (1994), "Criminometrics, latent Variables, panel data and different types of crime", Discussion Paper No. 124, Statistics Norway, Norway.

[2] Avio, K.L. y C.S. Clarke (1978), "The supply of property offences in Ontario: evidence on the deterrent effect of punishement", Canadian Journal of Economics, 11, pp. 1-19.

[3] Baltagi, B.H. (2001), Econometric Analysis of Panel Data, 2nd edition, John Wiley \& Sons. Chichester.

[4] Bandrés, E. y A. Díez-Ticio (2001), "Delincuencia y acción policial. Un enfoque económico", Revista de Economía Aplicada, 27, pp. 5-33.

[5] Barclay, G. y C. Tavares (2000), "International comparisons of criminal justice statistics 1998", Home Office Statistical Bulletin 04/00, Home Office, Research Development \& Statistics Directorate, London.

[6] Barclay, G. y C. Tavares (2002), "International comparisons of criminal justice statistics 2000”, Home Office Statistical Bulletin 05/02, Home Office, Research Development \& Statistics Directorate, London.

[7] Basmann, R. L. (1960), "On finite sample distributions of generalized classical linear identifiability test statistics", Journal of the American Statistical Association, 55, pp. 650-659.

[8] Becker, G.S. (1968), "Crime and punishment: An economic approach", Journal of Political Economy, 76, pp. 169-217.

[9] Block, M. K. y J.M. Heineke (1975), "A labor theoretic analysis of the criminal justice", American Economic Review, 65, pp. 314-325.

[10] Butcher, K.F. y A.M. Piehl (1998), "Cross-city evidence on the relationship between inmmigration and crime", Journal of Policy Analysis and Management, 17, pp. 457-493.

Revista Española de Investigación Criminológica

REIC AC 01-03 http:www.criminologia.net/revista 
[11] Cantor, D. y K.C. Land (1985), "Unemployment and crime rates in the post World-War II United States: A theoretical and empirical analysis", American Sociological Review, 50, pp. 317-332.

[12] Carr-Hill, R.A. y N.H. Stern (1973), "An economic model of the supply and control of recorded offences in England and Wales", Journal of Public Economics, 1, pp. 365-378.

[13] Cherry, T.L. y J.A. List (2002), “Aggregation bias in the economic model of crime", Economics Letters, 75, pp. 81-86.

[14] Chiricos, T.G. (1987), "Rates of crime and unemployment: An analysis of aggregate research evidence", Social Problems, 34, pp. 187-211.

[15] Cook, P. J. y G.A. Harkin (1985), "Crime and the business cycle”, Journal of Legal Studies, 14, pp. 115-128.

[16] Cornwell, C. y W. Trumbull (1994), "Estimating the economic model of crime with panel data", Review of Economics and Statistics, 76, pp. 360366.

[17] Dilulio, J. (1996), "Help wanted: Economists, crime and public policy", Journal of Economic Perspectives, 10, pp. 3-24.

[18] Eide, E. (2000), "Economics of Criminal Behavior", en Bouckaert and G. De Geests (eds.): Encyclopedia of Law and Economics, vol. V, Cheltenham, 2000, Edward Elgar, 345-389.

[19] Ehrlich, I. (1973), "Participation in illegitimate activities: A theoretical and empirical investigation", Journal of Political Economy, 81, pp. 521-567.

[20] Ehrlich, I. (1975), “On the relation between education and crime”. En: F.T. Juster, editor, Education Income and Human Behavior, chapter 12, Mc. Graw Hill Book Co, New York.

Revista Espa nola de Investigación Criminológica REIC AC 01-03 http:www.criminologia.net/revista 
[21] Entorf, H. y H. Spengler (2000), "Socieconomic and demographic factors of crime in Germany: Evidence from panel data of the German states", International Review of Law and Economics, 20, pp. 75-106.

[22] Entorf, H. y P. Winker (2001), “The economics of crime: Investigating the drugs-crime channel. Empirical evidence from the panel data of the German states”, Working Paper No. 2, International University of Germany, Bruchsel.

[23] Freeman, R.B. (1983), Crime and Unemployment, In: J.Q. Wilson (Ed.), Crime and Public Policy, London, ICS: San Francisco.

[24] García España, E. (2001), Inmigración y delincuencia en España: Análisis criminológico, Valencia, Tirant lo blanch.

[25] García España, E. y A.M. Prieto del Pino (1997), "Expulsión y detención de los inmigrantes ilegales en España. Ponencia, International Conference on the detention and explusion of illegal aliens, Tilburg, Holanda.

[26] Greenberg, D.F. (2001), "Time series analysis of crime rates”, Journal of Quantitative Criminology, 17, pp. 291-327.

[27] Hausman, J. (1978), "Specification tests in econometrics", Econometrica, 46, pp. 69-85.

[28] Hirschi, T. (1969), Causes of delinquency. University of California Press, Berkely (CA).

[29] Killias, M. (2001), Precis de criminologie, second edition, Berne, Staempfli.

[30] Levitt, S. (1996), "The effect of prison population size on crime rates: Evidence from prison overcrowding litigation", Quarterly Journal of Economics, 111, pp. 319-352.

[31] Levitt, S. (1997), "Using electoral cycles in police hiring to estimate the effect of police on crime", American Economic Review, 87, pp. 270-290.

Revista Española de Investigación Criminológica

REIC AC 01-03 http:www.criminologia.net/revista 
[32] Levitt, S. (1998), "Why do increased arrest rates appear to reduce crime: Deterrence, incapacitation, or measurement error", Economic inquiry, 36, pp. 353-372.

[33] Lochner, L. (1999), "Education, work and crime: Theory and evidence", Working Paper No. 465, Rochester Center for Economic Research, University of Rochester.

[34] Marselli, R. y M. Vannini (1997), "Estimating a crime equation in the presence of organized crime: Evidence from Italy", International Review of Law and Economics, 17, pp. 89-113.

[35] Marvell, T. y C. Moody (1991), "Age structure and crime rates: the conflicting evidence", Journal of Quantitative Criminology, 7, pp. 237-73.

[36] Marvell, T. y C. Moody (1996), "Specification problems, police levels and crime rates", Criminology, 34, pp. 609-646.

[37] Mas, M., Pérez, F. Uriel, U. Serrano, L. y A. Soler (2002): "Metodología para la estimación de las series de capital humano 1964-2001”, en: Capital Humano y Actividad Económica, Fundación Bancaixa, Valencia.

[38] Montero, A. y J. Torres (1998), La Economía del Delito y las Penas, Granada, Comares.

[39] Nagin, D. (1998), Criminal deterrence Research: A review of the evidence and a Research agenda for the outset of the 21st century, in Tonry, M. (ed) Crime and Justice: An annual review of Research, 23, pp. 1-42. University of Chicago Press.

[40] Papps, K. y R. Winkelmann (1998), "Unemployment and crime: New answers to and old question”, Discussion Paper No. 25, IZA, Bonn.

[41] Pastor, S. (1993), Ah! de la Justicia. Política Judicial y Economía, Madrid, Civitas.

Revista Española de Investigación Criminológica

REIC AC 01-03 http:www.criminologia.net/revista 
[42] Pastor, S. (1986), “Heroína y Política Criminal. Un Enfoque Alternativo”, en La Problemática de la Droga en España, Madrid, Edersa, pp. 225-256.

[43] Sala-i-Martin, X. (1997), "Transfers, social safety nets and economic growth”, International Monetary Fund Staff Papers, 44, pp. 81-102.

[44] Sandelin, B. y G. Skogh (1986), "Property crimes and the police: An empirical analysis of Swedish Municipalities", Scandinavian Journal of Economics, 88, pp. 547-561.

[45] Schmidt, P. y A. D. Witte (1984), An Economic Analysis of Crime and Justice: Theory, Methods, and Applications, New York: Academic Press.

[46] Small, J. y C. Lewis (1996), "Economic crime in New Zealand: Causation or coincidence?", Working Paper No. 158, University of Auckland.

[47] Smith, D.W., Devine, J.A. y J.F. Sheley (1992), "Crime and unemployment: Effects across age and race categories", Sociological Perspectives, 35, pp. $551-572$.

[48] Stageland, P. (2001), "Encuestas de Victimizacion”, en Díez-Ripollés, J.L. y A.I. Cerezo Domínguez (eds): Los problemas de la investigación empírica en criminología: La situación en España, Instituto Andaluz Interuniversitario de Criminología, Tirant lo Blanch.

[49] Trumbull, W. (1989), "Estimations of the economic model of crime using aggregate and individual level data", Southern Economic Journal, 56, pp. 423-439.

[50] Wahlroos, B. (1981), “On Finnish property criminality: An empirical analysis of the post-war era using Ehrlich model", Scandinavian Journal of Economics, 83, pp. 553-562.

[51] Witte, A.D. (1980), "Estimating the economic model of crime with individual data", Quarterly Journal of Economics, 94, pp. 57-84. 
[52] Wolpin, K.I. (1978), "An economic analysis of crime and punishment in England and Wales", Journal of Political Economy, 86, pp. 815-840.

Antonio Rodríguez Andrés ha obtenido Masters en económicas por la Universidad Carlos III de Madrid y la Université de Lausanne en Suiza. En la actualidad se encuentra completando sus estudios de doctorado en la Universidad de Odense (Dinamarca). Sus intereses de investigación se centran en el estudio de los aspectos econmicos de las actividades ilegales, la microeconometría aplicada y el estudio económico del derecho.

iD https://orcid.org/0000-0003-4111-1666

Revista Española de Investigación Criminológica REIC AC 01-03 http:www.criminologia.net/revista https://doi.org/10.46381/reic.v1i0.5 